\title{
LABORATORIO VIRTUAL EN LA FORMACION POR COMPETENCIAS
}

\section{VIRTUAL LABORATORY IN THE ABILITIES FORMATION}

Fecha de Recepción: 14 de Octubre de 2006

Fecha de Aprobación: 21 de Noviembre de 2006
Nelson Pérez ${ }^{1}$

Dario Amaya ${ }^{2}$

Olga Ramos ${ }^{3}$

RESUMEN: Una de las necesidades del país en la formación técnica, es la cobertura de mano de obra calificada en áreas técnicas, como la automatización de procesos industriales y, ahora con la globalización de los mercados, la industria requiere personal que apoye los procesos de modernización tecnológica que permitan implementar líneas de producción eficientes; para esto, es necesario contar con laboratorios especializados que permitan al individuo adquirir competencias en este campo. En este artículo se plantea el desarrollo de un laboratorio virtual que representa un proceso didáctico.

PALABRAS CLAVES: Laboratorio Virtual, socialización, Controlador de Lógica Programable, competencias laborales.

ABSTRACT: Nowadays, one of the most important requirements is qualified technical formation, like industrial automated processes. With the globalization phenomena, technological modernization appliances are demanded to implementate efficient production lines. This is supported in specialized laboratories that give the tools that allow to the student the appropriation of this practical knowledge. This article shows the development of a virtual laboratory and its automation application.

KEY WORDS: virtual laboratory, PLC, socialization of the knowledge

1 Universidad Distrital Francisco José de Caldas. Docente de postgrado.

2 Universidad Militar Nueva Granada. Docente de tiempo completo, Programa de Ingeniería Mecatrónica.

3 Universidad Manuel Beltrán. Coordinadora del laboratorio de Ingeniería Electrónica 


\section{INTRODUCCION}

El avance tecnológico en las áreas de la informática y las Comunicaciones (TIC), son actualmente una herramienta fundamental en el campo de la formación, ya que han permitido implementar recursos de apoyo académico como es el desarrollo de Laboratorios Virtuales (LV). Un LV consiste de aplicaciones de software que simulan procesos, instrumentos y/o elementos de medida, comparados con los laboratorios tradicionales; tienen una mayor flexibilidad y solucionan problemas de distancias, tiempo, espacios y recursos. Con la implementación de LV las instituciones educativas cuentan con espacios abiertos colaborativos que no solo permiten la integración de saberes, sino también el desarrollo de competencias y habilidades en determinados temas, para Carlos Ramos [1] son recursos que brindan oportunidad de formar comunidad para difundir y divulgar el conocimiento. Apoyados en las TIC, este documento hace referencia al diseño e implementación de un LV orientado a la formación en automatización industrial.

La puesta en marcha de este Laboratorio, busca crear un espacio virtual que llegue a mayor población, donde el estudiante pueda desarrollar las competencias pertinentes en el manejo y programación de señales discretas usando Controladores de Lógica Programable (PLC). Este desarrollo está realizado para un proceso didáctico mostrado en la figura 2. Este proceso está compuesto por dos manipuladores neumáticos de cuatro grados de libertad. El desarrollo cumple con una arquitectura de tres capas: la capa de presentación que constituye la comunicación entre el cliente y el sistema, es una aplicación JSP. La capa lógica, es quien interpreta y realiza peticiones a la base de datos, está implementada en Lenguaje de programación JAVA y para la parte gráfica de representación del proceso didáctico se utilizó JAVA 3D, la capa de persistencia es una base de datos que contiene la información correspondiente a los usuarios del sistema, esta implementada en MySQL.
En la sección II, se hace una breve descripción de la formación por competencias laborales y la interacción con el LV. En la sección III se presenta el desarroIlo del LV objeto de este documento.

\section{EL LABORATORIO VIRTUAL COMO APOYO A LA FORMACION POR COMPETENCIAS}

Las competencias, bajo el esquema de la formación, pueden ser competencias básicas o competencias laborales, según Miguel Maldonado [3], las primeras corresponden al sistema educativo y las segundas corresponden directamente a la educación orientada a la formación de mano de obra calificada. Una competencia laboral puede estar definida bajo ópticas diferentes, para Carlos Ramos [1], una competencia laboral es "el desarrollo de destrezas manuales y mentales básicas para el manejo de tecnologías específicas, conjuntamente en tres aspectos importantes que se manejan como bandera en la formación laboral de un individuo: saber, saber hacer y ser"; para Sergio Tobón [2], "las competencias son procesos a través de los cuales resuelven problemas del contexto laboral articulando el saber hacer, el saber conocer y el saber ser". Se puede concluir que una competencia es un procedimiento que busca integrar los conocimientos adquiridos, la destreza motriz y las actitudes y valores del individuo para fortalecer su desarrollo personal y laboral.

Los LV son un apoyo para la formación por competencias pues se tienen recursos virtuales sin dificultades de espacio, lo que permite que las prácticas se puedan realizar, en el momento que se desee en el lugar de formación, en centros pilotos [4], o desde sus hogares.

El LV desarrollado abre la posibilidad de que el estudiante desarrolle las siguientes, competencias y habilidades:

- Programación de PLC.

- Diseño e implementación de algoritmos que per- 
mitan el óptimo desempeño de un sistema en el manejo de variables discretas.

- Diseño e implementación de estrategias de control.

- Desarrollo de trabajo en equipo

- Planeación y organización del trabajo.

- Destreza en el manejo de herramientas informáticas.

\section{DESARROLLO DEL LABORATORIO VIRTUAL}

El desarrollo descrito a continuación está orientado a la automatización de procesos industriales. La programación del PLC se ha desarrollado en lenguaje ladder. La arquitectura del desarrollo corresponde a un modelo de tres capas como se muestra en la figura 1.

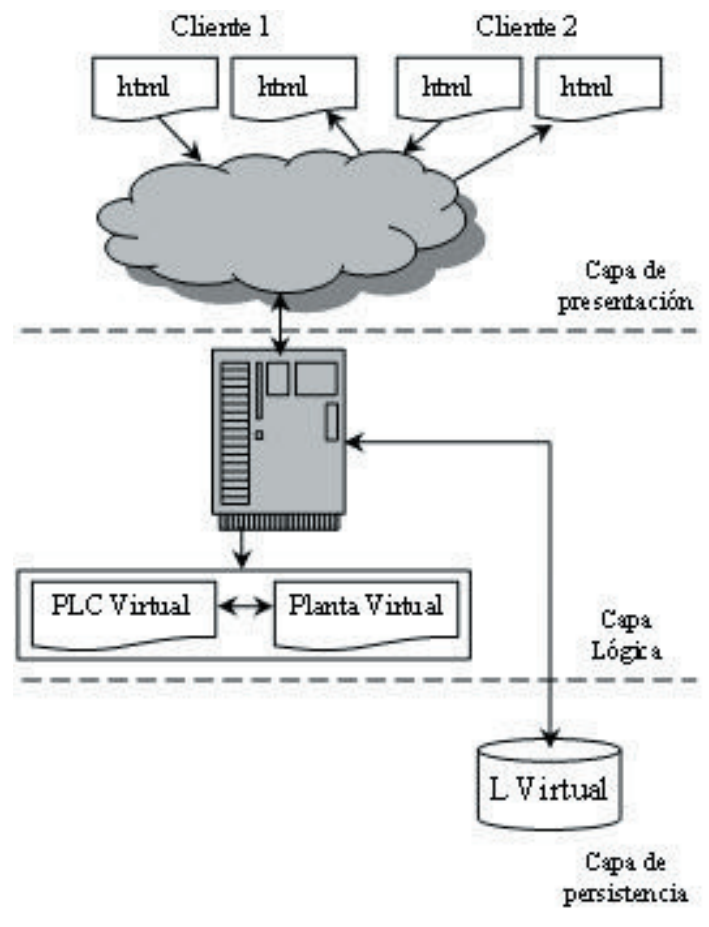

Figura 1. Estructura general
En la capa de presentación se implementaron los formularios de acceso al sistema de información. Desde allí, el usuario puede hacer su validación, como administrador o como estudiante, a través de la conexión que realiza desde cualquier navegador de Internet y acceder al servidor de aplicaciones Web. Para este caso se utilizó Jboss ${ }^{4}$. Una vez establecida la conexión, se solicita al usuario la contraseña respectiva; si se valida como administrador puede inscribir estudiantes, profesores, asignaturas e instituciones que son usuarias del sistema; si lo hace como estudiante, es llevado a un applet de la planta virtual, y a través de éste navegar al PLC virtual.

En la capa lógica se desarrollaron los algoritmos para representar el proceso. Se implementó la clase Estacion20, usando API java3D, se creó la estructura del proceso que se muestra en la figura 2.

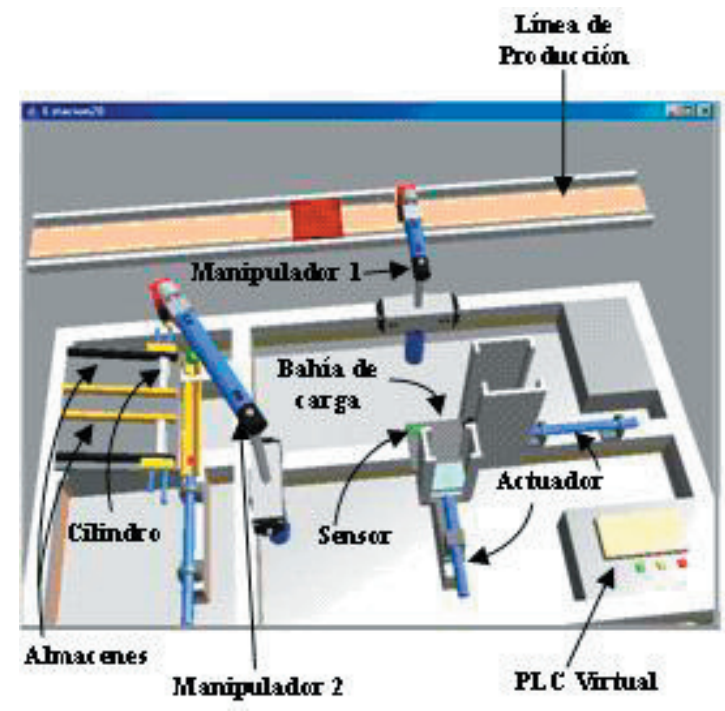

Figura 2. Planta Virtual

La planta está compuesta por una línea de producción que es alimentada con materia prima, representada por dos cilindros, a través del manipulador 2, que toma las piezas desde los almacenes y las lle-

4 Servidor de aplicaciones para desarrollos Java con licenciamiento libre que puede funcionar bajo cualquier sistema operativo que lo soporte 
va a la bahía de carga, allí las deposita el manipulador 1. Los manipuladores manejan cuatro grados de libertad: movimiento vertical, movimiento horizontal, movimiento rotacional y abren y cierran pinzas de agarre propios de cada manipulador. La parte de control está a cargo de un PLC virtual que maneja hasta 255 sensores virtuales o entradas e igual cantidad de actuadores o salidas. Las señales de entrada y de salida son digitales.

Para el movimiento de cada uno de los elementos se construyó la clase Movimiento, que permite implementar los métodos necesarios para esta actividad. Los métodos de la clase movimiento reciben y devuelven parámetros de la clase compilador, es decir activan o desactivan señales discretas, de acuerdo al algoritmo desarrollado por el estudiante. La clase Compilador representa el PLC virtual y contiene el compilador en lenguaje Ladder que permite al usuario realizar los algoritmos que aplicará al proceso. La figura 3, muestra la ventana que despliega ésta clase.

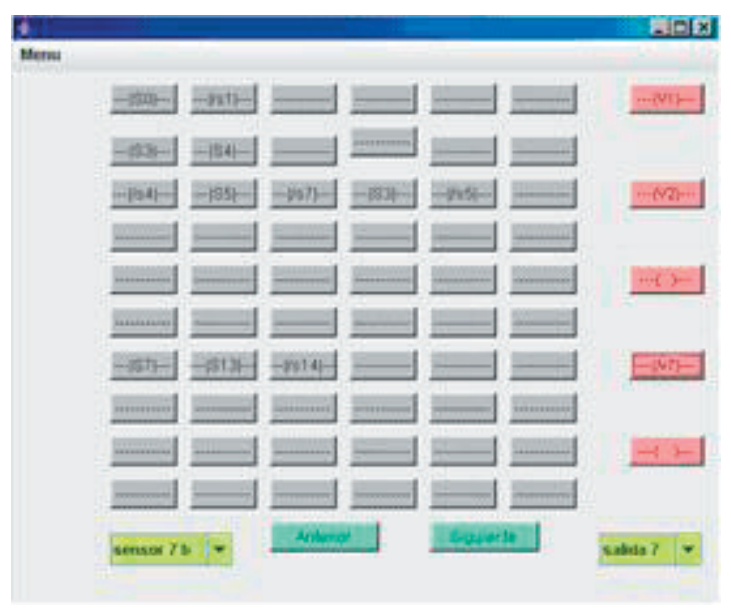

Figura 3. Compilador

Luego de la programación realizada por el estudiante, él puede compilar y después ejecutar, a través de un menú, el programa, observando de una vez los resultados del algoritmo implementado.

La capa de persistencia está desarrollada en Mysql.
La base de datos se llama LVirtual y contiene un sistema de información donde se encuentra los datos pertinente al administrador, los usuarios, las entidades y las asignaturas que pueden acceder al Laboratorio.

\section{CONCLUSIONES}

Esta aplicación, permite la expansión en cobertura de la educación, logrando socializar el conocimiento.

EI LV desarrollado da la oportunidad al usuario de realizar las prácticas sin la restricción de tiempo que si tendría en un laboratorio real, permitiendo ejercitar y así tener la posibilidad de adquirir las competencias necesarias par luego enfrentar un equipo real o un proyecto completo de automatización industrial.

La realización de este tipo de proyecto, genera una solución para las entidades de educación en la implementación de sus Laboratorios ya que sistemas de difícil adquisición por su costo o por su alta complejidad se pueden elaborar a través de esta forma.

\section{REFERENCIAS}

[1] RAMOS, C. O. Estudio de una Plataforma para Formación Técnica Virtual en el SENA- Modelo y Arquitectura de la Red. Bogotá, 2002. Trabajo de grado (Magíster en Teleinformática). Universidad Distrital Francisco José de Caldas. Facultad de Ingeniería. Maestría en Teleinformática.

[2] TOBÓN, S. Formación Basada en Competencias. Ecoe Ediciones. Bogotá junio de 2004.

[3] MALDONADO GARCÍA M. A. Las competencias una opción de vida. Ecoe Ediciones. Bogotá Noviembre de 2003.

[4] PÉREZ, J. GUILLEN, E. SEPÚLVEDA, N. RAMOS, C. O. Platfform of Virtual Training for Work. Springer Berlin, vol. 2722/2003, pag.154-157. 